University of Louisville

ThinkIR: The University of Louisville's Institutional Repository

\title{
Examining the effects of saccade execution and handedness on proactive interference.
}

Montgomery A. Kroger

University of Louisville

Follow this and additional works at: https://ir.library.louisville.edu/honors

Part of the Cognitive Psychology Commons

\section{Recommended Citation}

Kroger, Montgomery A., "Examining the effects of saccade execution and handedness on proactive interference." (2014). College of Arts \& Sciences Senior Honors Theses. Paper 84.

http://doi.org/10.18297/honors/84

This Senior Honors Thesis is brought to you for free and open access by the College of Arts \& Sciences at ThinkIR: The University of Louisville's Institutional Repository. It has been accepted for inclusion in College of Arts \& Sciences Senior Honors Theses by an authorized administrator of ThinkIR: The University of Louisville's Institutional Repository. This title appears here courtesy of the author, who has retained all other copyrights. For more information, please contact thinkir@louisville.edu. 
Examining the Effects of Saccade Execution and Handedness on Proactive Interference

\section{By}

\section{Montgomery A. Kroger}

Submitted in partial fulfillment of the requirements for Graduation summa cum laude and

for Graduation with Honors from the Department of Psychology

University of Louisville

March 2014 


\begin{abstract}
Proactive interference is the phenomenon by which previously-learned information impairs recall of more recently-learned information. The present experiment was conducted to investigate two factors that may affect the occurrence of proactive interference. The first of these factors is saccade execution. Saccade execution has been shown in numerous studies to improve performance on a wide range of memory tasks. The second factor was people’s consistency of handedness, a term referring to the strength of people's tendency to use one hand (right or left) over the other. Although the results of the primary analyses were inconclusive, further exploration of the data led to the proposition that saccades increase memory performance when no interference effect is induced, but may reduce performance when there is an interference effect. These results, although not significant in the expected way, still showed hints of interesting results.
\end{abstract}

Keywords: handedness, proactive interference, paired associate recall, memory 
Examining the Effects of Saccade Execution and Handedness on Proactive Interference Proactive interference, which was originally coined the “priority effect” (Tulving \& Watkins, 1974), has long been thought to be a reason for memory loss and forgetfulness. Proactive interference is the phenomenon where memory is impaired in the present because of information learned in the past. In an early demonstration, Tulving and Watkins showed participants word-pairs (e.g., plow-sock) on a first list and later showed them word-pairs on a second list. The cue word, or the first word, in each pair on the second list was either the same cue word in a pair on the first list (e.g. plow-name) or different (e.g., hip-name). On a test, participants were given a cue word and asked which word, or target word, was paired with it on the second list (e.g., plow-?). Proactive interference manifested as participants' reduced ability to recall target words from the second list if a pair with the same cue word had previously been learned (i.e., lower recall of name from the pair plow-name than the pair hip-name). In this paradigm, pairs for which there is a potential source of interference in the form of a previously learned pair with the same cue word are called "interference pairs”. In contrast, control pairs are those for which no pair with the same cue word was previously learned. For example, if participants studied two completely unrelated word-pairs (e.g., plow-sock and hip-name), and then were tested on hip-?, memory for sock cannot interfere with recalling name. In everyday life, proactive interference can be seen in people’s difficulty remembering a new locker combination because of an old one, or mistakenly calling a new doctor by an old doctor's name. In each case, the two target pieces of information (a locker code and a name) are bound to the same cue (a locker and a relationship), making it difficult to recall the new information. The AB, A-C paradigm is the experimental design developed from the Tulving and Watkins’s experiment, and this paradigm was implemented in this study as well. 
The execution of saccadic eye movements has recently come to light as a possible means of memory enhancement. Numerous tests have shown that executing horizontal saccades has improved performance on a variety of memory tests. Horizontal saccade execution, as implemented in these studies, is the repetitive movement of the eyes back and forth to follow a dot flashing left of center and right of center on a computer screen. The performance of participants who execute saccades is most often compared to the performance of participants in a fixation condition, in which participants keep their eyes fixed on a dot that flashes in the center of the screen without changing position. Saccades have helped participants perform in free recall tasks (Lyle, Logan, \& Roediger, 2008, Experiment 1), in which participants in the saccade condition were more likely than participants in the fixation condition to remember, in any order, words studied earlier. Another example is old/new recognition (Parker, Relph, \& Dagnall, 2007), in which participants study a list of items and then are shown a test list and asked whether items on the test list were on the studied list (old) or not (new). Participants who performed saccades were more accurate in recognizing which words they had seen, and also less likely to falsely claim to have seen new words. A third example is associative recognition (Brunyé, Mahoney, Augustyn, \& Taylor, 2009), in which, after studying multiple maps, participants were presented with maps and asked whether each was identical to the ones studied earlier or had been slightly altered. Possible alterations included the addition or removal of objects within the map. Participants after saccades were less likely to claim that the map was the same when it had in fact been altered (see also Lyle, Hanaver-Torrez, Häcklander, \& Edlin, 2012; Parker et al., 2008, Experiment 1). The effect whereby saccade execution improves memory is called saccadeinduced retrieval enhancement (SIRE). 
Although saccades often have a positive effect on the ability to retrieve various pieces of information, it is unclear exactly how saccades produce this effect. It has been proposed (Lyle \& Edlin, in press) that saccades bring to the foreground of participants’ memory previously learned information, and will especially help when confronted with conflict, such as similar cues. In other words, saccades may prioritize or privilege recently-learned information. Alternatively, saccades may inhibit information that is not relevant. It is also possible that saccades simultaneously privilege recent information and inhibit irrelevant information.

Another factor in memory is consistency of handedness. Consistent-handed individuals use one hand almost exclusively, while inconsistent-handed people are more likely to use their hands interchangeably for different tasks. In recent studies, consistency of handedness has been shown to be a predictor of performance on various memory tasks. Inconsistent-handed people have shown the tendency across a variety of memory tasks to retrieve memories more accurately than consistent-handed people. For example, inconsistents are less likely to falsely identify a rearranged word pair as one that was previously studied (Lyle et al., 2012) and less likely to recall words that were not studied (Christman, Propper, \& Dion, 2004). They are also less likely to state that false information is true after receiving with misleading information (Lyle \& Jacobs, 2010). Their advantage also extends to other tasks. They are less likely to misidentify briefly flashed faces of unknown individuals as famous (Lyle \& Osborn, 2011), more likely to recall details of their own lives (Parker et al., 2008), and better able to accurately recall words from studied lists (Propper, Christman, \& Phaneuf, 2005). In sum, inconsistent-handed people, under normal circumstances, tend to remember more accurately. However, studies have indicated that, while saccades improve memory retrieval for consistent-handed people, they sometimes negatively impact inconsistent-handed people. Inconsistents recalled more incorrect responses 
on a free recall test after saccades than after fixation (Lyle et al., 2008), and claimed to recognize word-pairs from a studied list even though the word-pairs had been changed (Lyle et al. 2012).

The effect of saccade execution on proactive interference has never been studied. This study was designed to show that saccades help bring to the foreground of attention desired information, especially when confronted with conflicting or interfering memories. The first hypothesis was that all participants would have better memory for control word-pairs than for interference word-pairs in A-B, A-C paradigm (Tulving \& Watkins, 1974). The second hypothesis was that inconsistents overall would recall more words than consistents. Based on recent research regarding saccades, the third hypothesis was that saccades would be beneficial primarily to consistent-handed individuals. In other words, consistents who performed saccades would recall more than those who performed the fixation activity. The fourth hypothesis was that saccades would improve recall for interference word-pairs more than they would improve recall for control word-pairs, given the theory that saccades improve performance primarily when there is conflict in memory (Lyle \& Edlin, in press). Conflict, in the form of proactive interference, exists in the interference condition but not the control condition. The final hypothesis was that inconsistent-handed participants in the saccades condition would show less improvement than consistent-handed participants in the saccades condition or inconsistenthanded participants who did not perform saccades.

\section{Method}

\section{Participants}

Participants were 62 University of Louisville students enrolled in psychology course (43 female, 19 male) who were recruited using the SONA research participation system and ranged in age from 18 to 30 years $(M=19.8$ years). Participants received course credit for participating. 
Thirty-nine participants were classified as consistent-handed and 23 as inconsistent-handed (the process of classification is explained in Materials and Procedure).

\section{Materials}

Handedness was assessed using a modified version of Oldfield’s (1971) Edinburgh Handedness Inventory (see Lyle, McCabe, \& Roediger, 2008, for details) which yields scores ranging from -100 (exclusive left-handedness) to +100 (exclusive right-handedness). In two previous samples of undergraduates (Lyle et al., 2008), the median score was +80 and individuals who scored +80 or higher were affected differently by saccades than individuals who scored lower. Therefore, in this study, participants were classified as consistent if they scored at or above +80 or at or below -80 . Participants were inconsistent if they scored between +80 and 80.

From the English Lexicon Project (Balotta, Yap, Cortese, Hutchison, Kessler, Loftis, Neely, Nelson, Simpson, \& Treiman, 2007), 164 words were randomly selected. Each word was three to five letters in length, and all were singular nouns with a HAL (Hyperspace Analogue to Language) Frequency of at least 140. HAL Frequency is an estimate of how often a word appears in the English language. Two lists of 40 word-pairs each were developed. In List 1, all 40 of the word-pairs were created by randomly pairing two words. In List 2, 20 of the wordpairs were created by randomly pairing two words. These served as control pairs in the experiment. For the other 20 word-pairs on List 2, the first word, or cue word, was copied from a word-pair in List 1 . These words from List 1 were then paired with randomly-chosen second words, or target words. These served as interference pairs in the experiment. Consequently, 20 of the word-pairs in List 2 were completely original (i.e., neither word had appeared in List 1), while 20 of them had an original target word but a repeated cue word from List 1 word-pairs. 
For reasons explained below, the researcher made certain that, for interference pairs, the target word in each list did not start with the same letter. A representative list of word-pairs is represented in Figure 1, with the interference word-pairs italicized.

Figure 1

\begin{tabular}{|c|c|}
\hline List 1 & List 2 \\
\hline tile - chest & dock - bet \\
poppy - shade & rise - worm \\
lock - storm & lock - knot \\
decoy - brow & decoy - reel \\
\hline
\end{tabular}

The order of Lists 1 and 2 was counterbalanced, meaning that approximately half the participants studied them in one order and the other participants studied them in the opposite order. The two different orders were arbitrarily labeled version A and version B. Participants were randomly assigned to receive one version or the other.

In studies like these, it is important to minimize primacy and recency effects, whereby people tend to remember the first few items and last few items in a list. Therefore, three wordpairs were placed at the beginning and end of each list. Memory for these items was not tested.

The stimulus for the saccades task was a computerized sequence showing a black circle on a white background. The circle alternated between $13.5^{\circ}$ left and $13.5^{\circ}$ right of the vertical midline every $500 \mathrm{~ms}$ for $30 \mathrm{~s}$. For the fixation task, the circle flashed in the center of the screen (500 ms on, $500 \mathrm{~ms}$ off) for $30 \mathrm{~s}$.

\section{Procedure}


Up to five individuals participated at the same time. Participants gave informed consent, then completed a modified paper-and-pencil handedness inventory and reported their sex and age. After these were collected, participants were randomly assigned to one of four conditions. These conditions were created by fully crossing the pretest activity (fixation or saccades) with version (A or B). Participants were then read aloud the instructions for the first list. They were told that they would be shown a word-pair and asked to relate the two words in their minds. After eight seconds, a screen would automatically appear, asking them to rate how successful they were at relating the two words on a scale from one (not successful) to five (very successful). They were told that they might be asked to recall the words later. They were also told that, if they were asked to remember the words, they would be given the first word and asked to recall the second. Participants were then asked if they had any questions, and informed that it was important that they understand. After any questions were answered, they were told that pressing “c” would start the study, and the experimenter left the room.

Participants were then shown a word-pair, written in either green or yellow on a black background. After eight seconds, they were told to type a number from one to five rating their success in creating a connection. This process was repeated 46 times, counting three buffer pairs placed at the beginning and the end to counteract primacy and recency effects, and then participants were told to take a two-minute break. After this break, participants were shown another screen, which told them the same instructions as they had been given for the first list. These words appeared in whichever color participants had not seen on the first list. That is, if the first list had been green, the second list was yellow, and vice versa. They were again shown 46 word-pairs and asked after each to rate their success at relating the two words. After the second list, they were told to take another two-minute break. 
After this break, participants were shown a screen explaining the pretest activity they would perform. In the fixation activity, participants were told to keep their eyes focused on the blinking black dot in the middle of the screen and not move their eyes until told that it was okay for them to do so. In the saccades activity, participants were told to keep their eyes focused on the blinking black dot that alternated between two positions on the left and right side of the screen. Participants were told to maintain focus on the dot by moving their eyes only (i.e., without moving their head). The dot blinked for 30 seconds in each condition, during which time the experimenter watched to make certain that participants were performing the task. The experimenter did not enter the room so as not to distract participants who were in other parts of the study.

After the pretest activity, participants received instructions for the memory test. They were told that the screen would show a word that had been the first word in a word-pair they had studied. Their task was to recall the second word from each word-pair in the second list. They were told that some words had served as the first word on both lists, and it was made clear that their task was to recall the second word from the second list. To help them remember, the instructions told them what color the second list had been (whether green on black or yellow on black). Participants were given the first letter of the second word so that, when tested on interference pairs, they would not accidentally recall a word from the first list (as described above, the second word in interference pairs started with a different letter on each list). They were told to type the whole word, not simply the letters after the first one. Although some participants did not follow this instruction, the researcher gave credit when scoring the recall data to participants who either spelled out the whole word or only included the letters after the first. The rationale was that subjects who only included the later letters nonetheless knew what 
the answer was, but had simply not followed the directions appropriately. Figure 2 depicts the procedure visually.

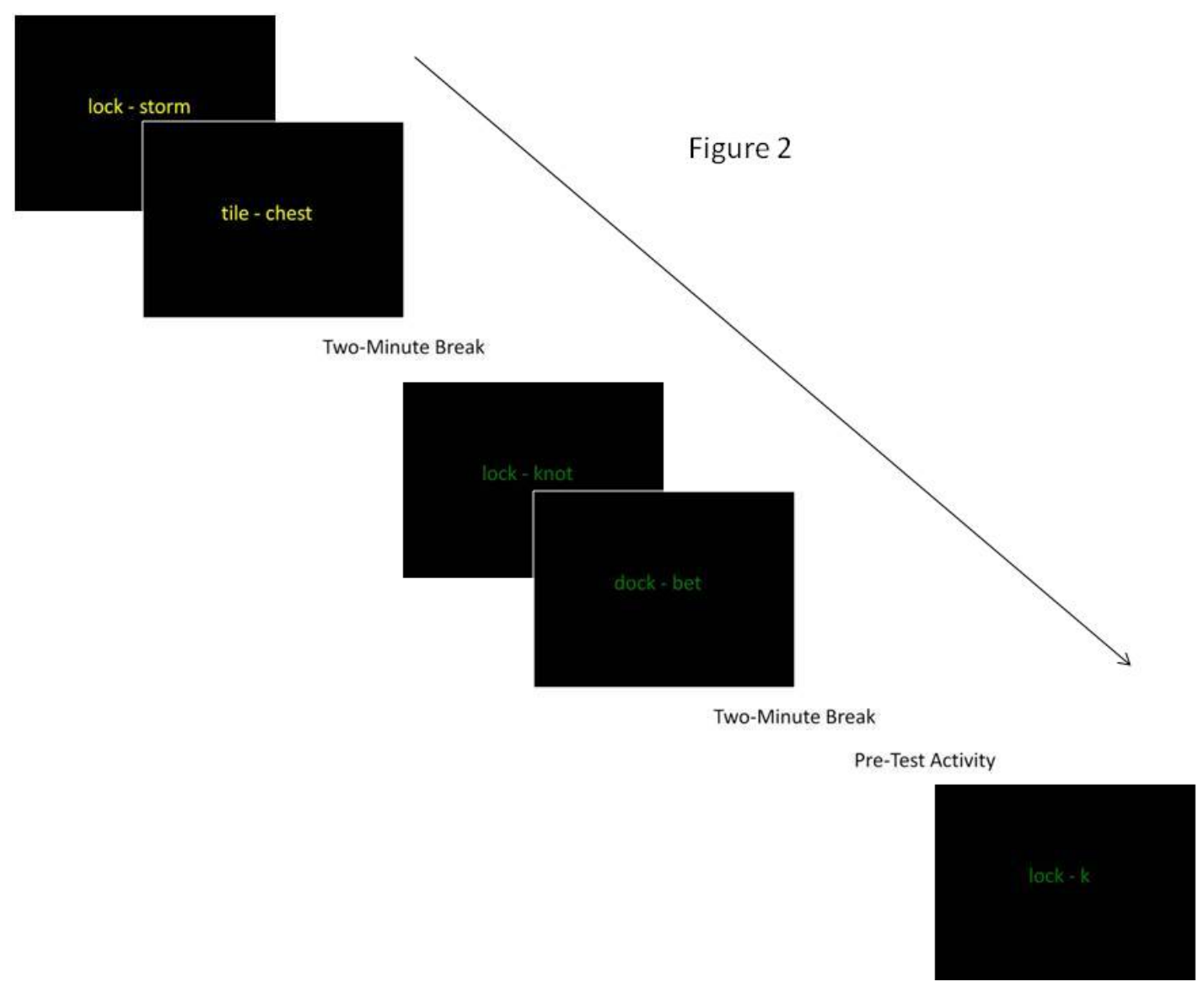

After the test, the program immediately proceeded to a debriefing screen. The objective of the study (i.e., to study saccade execution, handedness, and proactive interference) was explained to participants, who were thanked for their time and dismissed.

\section{Data Analysis}

The experiment had a 2 (handedness: inconsistent versus consistent) X 2 (pretest activity: fixation versus saccades) X 2 (word-pair type: control versus interference) mixed design, where the first two factors were between-subjects and the third was within-subjects. These data were 
submitted to a 2 X 2 X 2 factorial ANOVA, and the dependent variable was proportion of words correctly recalled.

\section{Results}

Although some patterns were in the expected direction, there were no significant effects. The main effect of item type was not significant, $F(1,58)=1.475, p=.229$, although the proportion correct for interference pairs was, as expected, lower $(M=.427)$ than for control pairs $(M=.450)$. The main effect of handedness was not significant, either, $F(1,58)=1.796, p=.185$. Again, though, proportion correct was higher for inconsistent participants than consistent ones (Ms $=.475$ and .402 respectively). The main effect of activity was not significant, $F(1,58)=$ $.009, p=.923$, but proportion correct for participants who performed saccades was slightly higher $(M=.441)$ than for those who performed fixation $(M=.436)$. There was no two-way interaction between item type and activity, $F(1,58)=.004, p=.952$, or three-way interaction between item type, activity, and handedness, $F(1,58)=.063, p=.802$.

For exploratory purposes, the primary ANOVA was rerun with the factor of version included. When included, there were two significant three-way interactions. The first was activity by handedness by version, $F(1,54)=8.801, p=.006$. Looking first at consistenthanders, those who performed saccades before testing in version B performed better than those who performed fixation in the same version. In contrast, in version A, saccades negatively impacted consistent-handers compared to fixation. The opposite holds true for inconsistenthanders, with saccades appearing to help them in version A, but hurt them in version $\mathrm{B}$. The means are shown in Figure 3. 


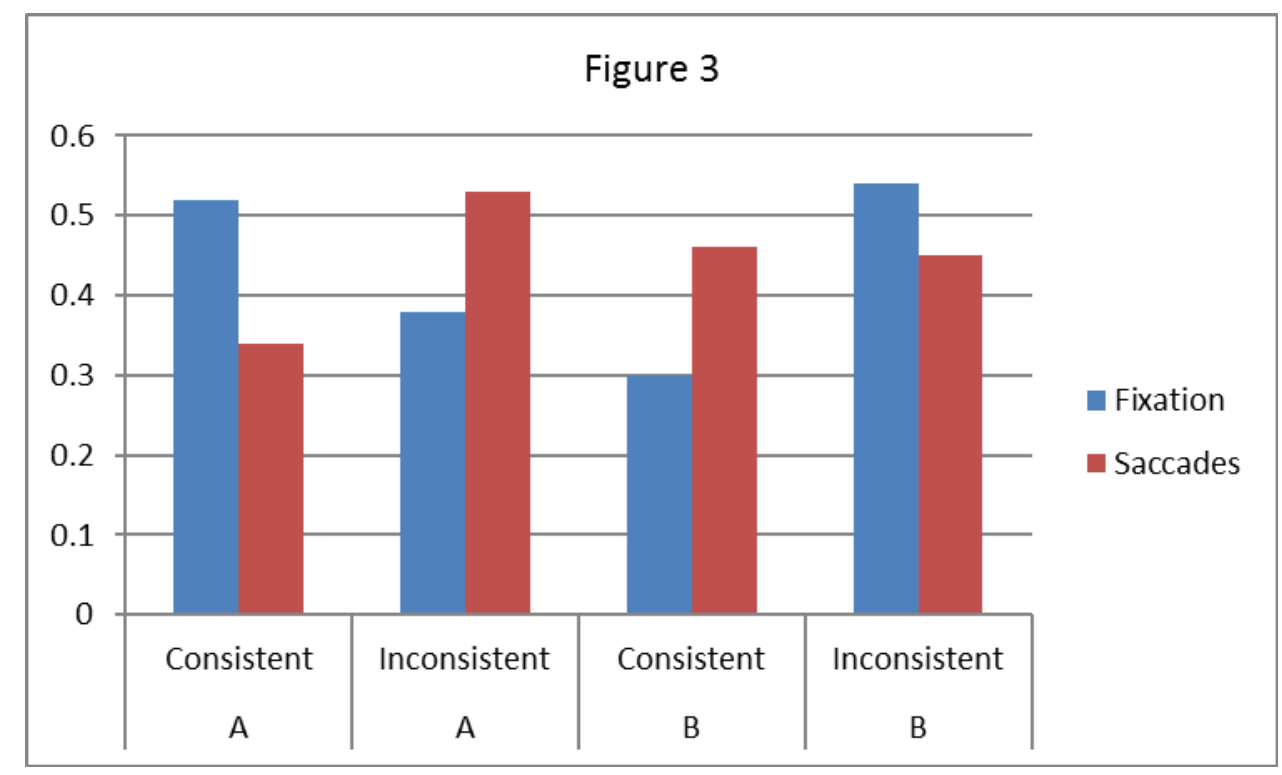

An interesting pattern also emerged in a significant version by handedness by item type interaction, $F(1,54)=4.461, p=.039$. Again looking at consistent-handers first, there was no evidence of proactive interference in version $\mathrm{B}$, but there was in version $\mathrm{A}$. The opposite is true for inconsistent-handers, who showed no hint of interference in version A, but did in version B. The means are shown in Figure 4.

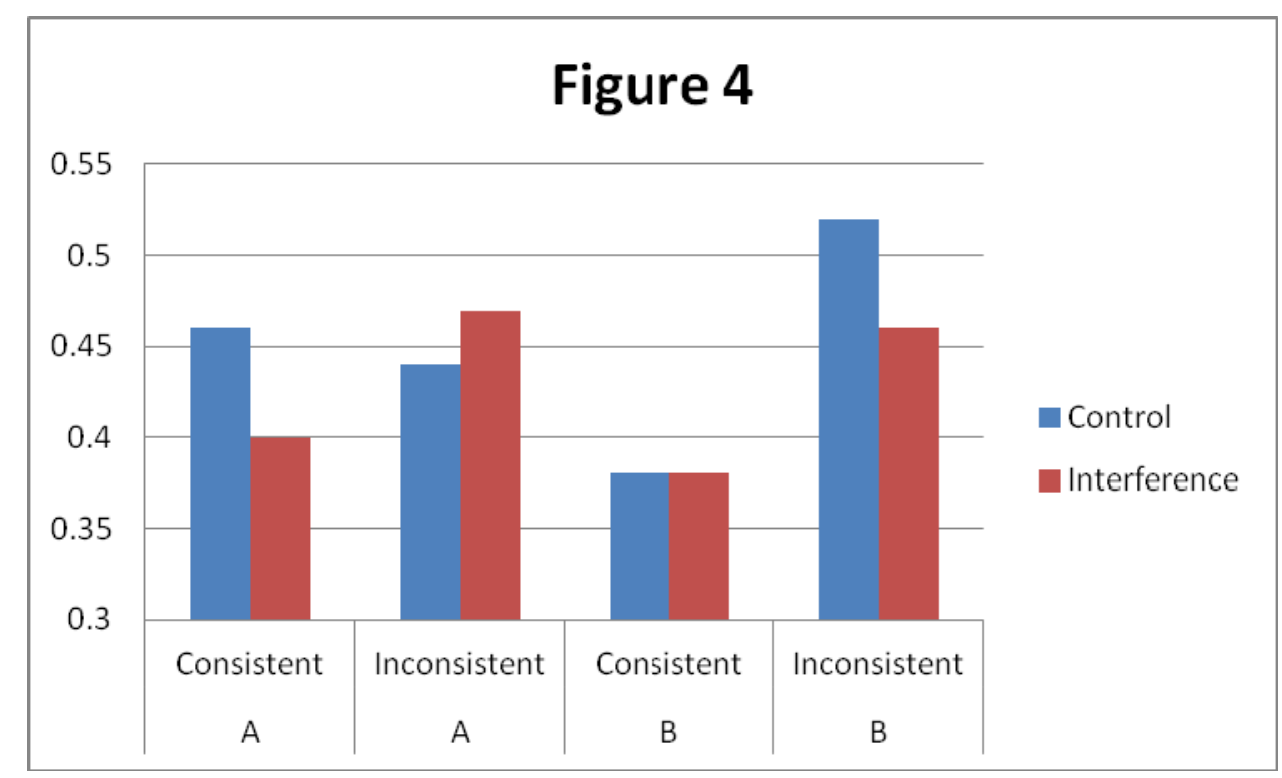




\section{Discussion}

Initially the results would appear to be inconclusive. While there was a trend toward an interference effect, it was not conventionally significant. Also, inconsistent-handed participants did numerically better than consistent-handed participants, but the effect was not significant. Finally, participants who performed saccades had numerically higher recall than those who performed fixation, but the difference was miniscule and non-significant. Two factors may have contributed to these unexpectedly weak results. First, when searching for a handedness-based memory difference, it is preferable to have a larger sample size than in the current experiment. Ideally, the sample size would have been closer to 100, but a slow rate of participant signups prevented this. Second, it is possible that participants may have had trouble forming associations between word-pairs on the first list. If participants were unable to create this connection, it is unlikely there would be a robust interference effect, because the memory from the first list would not come to mind when trying to recall memories of the second list. A way of investigating this possibility would be to look at the ratings participants made on the first list. After each wordpair had been presented for eight seconds, participants were asked to rate how successful they were at relating the two words on a scale from one (not successful) to five (very successful). If their ratings were low in the first list, an interference effect would be unlikely. Whether participants had higher ratings for the second list or not, their low ratings for the first list would indicate that those word-pairs would not be sufficiently well-remembered to create interference.

On the other hand, the results of the exploratory analysis, including version as a variable, seem to indicate that for some conditions there was a significant interference effect. For two groups, (inconsistents in version A and consistents in version B), there was no hint of interference. Indeed, the means show that participants actually performed slightly worse on the 
control pairs than the interference pairs. However, in those two conditions, saccades increased memory performance overall. In contrast, for inconsistents in version B and consistents in version A, there was evidence of an interference effect, but saccades reduced memory performance.

This study was initiated with the expectation that saccades would help participants recall the word-pair that they wanted to recall. In this procedure, the desired word-pairs were the ones from the second list. However, in those versions in which there was evidence of proactive interference, saccade execution made it harder to recall the word from the second list, perhaps because saccade execution was actually increasing recall of the word from the first list. In those versions in which there was no evidence of interference, saccades increased recall of words from the second list, as would be expected from a typical SIRE effect.

These results suggest a need for further study. In a follow-up experiment, there could be a condition in which word-pairs on List 1 were considerably easier to relate than word-pairs on List 2. For example, if a word-pair in List 1 was fire-truck, a word pair in List 2 could be firelock. The prediction would be that, in this condition, a robust interference effect would occur because the first word-pair is easier to relate than the second word-pair based on preexisting associations. Furthermore, saccades would significantly reduce recall of words from List 2 (e.g., lock), because they would improve recall for the words already better remembered. In another condition, List 1 would contain the difficult-to-associate word-pairs and List 2 the easy ones. This, presumably, would lead to no interference effect. In that condition, saccades execution should increase recall of words from List 2. 
While this experiment did not find the results it was initially seeking, two factors may have affected this. The first of these is sample size, since signups were so low. The other potential factor was the nature of the word-pairs. If certain word-pairs were harder to relate, it makes sense that no interference effect would occur, thus somewhat undermining the findings. However, these data do seem to indicate that saccades are not necessarily beneficial in interference-related tasks. The idea that saccades increase the power of the word-pair that is already better-remembered is one that has not been shown in previous research, and warrants further inquiry. This could lead to a greater understanding of what exactly it is that saccades do, and how that could be beneficial or detrimental in the future. 


\section{References}

Balotta, D. V., Yap, M. J., Cortese, M. J., Hutchison, Keith. A., Kessler, B., Loftis, B., Neely, J. H., Nelson, D. L., Simpson, G. B., \& Treiman, R. (2007). The English Lexicon Project. Behavior Research Methods, 39(3), 445-459.

Brunyé, T. T., Mahoney, C. R., Augustyn, J. S., \& Taylor, H. A. (2009). Horizontal saccadic eye movements enhance retrieval of landmark shape and location information. Brain and Cognition, 70, 279-288.

Christman, S. D., Propper, R. E., \& Dion, A. (2004). Increased interhemispheric interaction is associated with decreased false memories in a verbal converging semantic associates paradigm. Brain and Cognition, 56, 313-319.

Lyle, K. B., \& Edlin, J. M. (in press). Why does saccade execution increase episodic memory retrieval? A test of the top-down attentional control hypothesis. Memory.

Lyle, K. B., Hanaver-Torrez, S. D., Hackländer, \& Edlin, J. M. (2012). Consistency of handedness, regardless of direction, predicts baseline memory accuracy and potential for memory enhancement. Journal of Experimental Psychology: Learning, Memory, and Cognition, 38, 187-193.

Lyle, K. B., \& Jacobs, N. E. (2010). Is saccade-induced retrieval enhancement a potential means of improving eyewitness evidence? Memory, 18, 581-594.

Lyle, K. B., Logan, J. M., \& Roediger, H. L., III. (2008). Eye movements enhance memory for individuals who are strongly right-handed and harm it for individuals who are not. Psychonomic Bulletin \& Review, 15, 515-520. 
Lyle, K. B., McCabe, D. P., \& \& Roediger, H. L., III. (2008). Handedness is related to memory via hemispheric interaction: Evidence from paired associate recall and source memory tasks. Neuropsychology, 22(4), 523-530.

Lyle, K. B., \& Orsborn, A. E. (2011). Inconsistent handedness and saccade execution benefit face memory without affecting interhemispheric interaction. Memory, 19, 613-624.

Parker, A., Relph, S., \& Dagnall, N. (2008). Effects of bilateral eye movements on the retrieval of item, associative, and contextual information. Neuropsychology, 22, 136-145.

Propper, R. E., Christman, S. D., \& Phaneuf, K. A. (2005). A mixed-handed advantage in episodic memory: A possible role of interhemispheric interaction. Memory \& Cognition, 33, 751-757.

Tulving, E., \& Watkins, M. J. (1974). On negative transfer: Effects of testing one list on the recall of another. Journal of Verbal Learning and Verbal Behavior, 13, 181-193. 